\title{
Supplementary Material for "Room temperature hyperpolarization of polycrystalline samples with optically polarized triplet electrons: Pentacene or Nitrogen-Vacancy center in diamond?"
}

Koichiro Miyanishi ${ }^{1}$, Takuya F. Segawa ${ }^{2,3}$, Kazuyuki Takeda ${ }^{4}$, Izuru Ohki ${ }^{5}$, Shinobu Onoda ${ }^{6,7}$, Takeshi Ohshima $^{6,7}$, Hiroshi Abe ${ }^{6,7}$, Hideaki Takashima ${ }^{8}$, Shigeki Takeuchi ${ }^{8}$, Alexander I. Shames ${ }^{9}$, Kohki Morita $^{5}$, Yu Wang ${ }^{4}$, Frederick T.-K. So ${ }^{2,6}$, Daiki Terada $^{2,6}$, Ryuji Igarashi ${ }^{6,7,10}$, Akinori Kagawa $^{1,10,11}$, Masahiro Kitagawa ${ }^{1,11}$, Norikazu Mizuochi ${ }^{5}$, Masahiro Shirakawa ${ }^{2,6}$, and Makoto Negoro $6,10,11$

${ }^{1}$ Graduate School of Engineering Science, Osaka University, Toyonaka, Osaka 560-8531, Japan

${ }^{2}$ Department of Molecular Engineering, Graduate School of Engineering, Kyoto University, Nishikyo-Ku, Kyoto 615-8510, Japan

${ }^{3}$ Laboratory for Solid State Physics, ETH Zurich, 8093 Zurich, Switzerland

${ }^{4}$ Division of Chemistry, Graduate School of Science, Kyoto University, Kyoto 606-8502, Japan

${ }^{5}$ Institute for Chemical Research, Kyoto University, Gokasho, Uji, Kyoto, 611-0011, Japan

${ }^{6}$ Institute for Quantum Life Science, National Institutes for Quantum and Radiological Science and Technology, 4-9-1, Anagawa, Inage-Ku, Chiba 263-8555, Japan

${ }^{7}$ Takasaki Advanced Radiation Research Institute, National Institutes for Quantum and Radiological Science and Technology, 1233 Watanuki, Takasaki, Gunma 370-1292, Japan

${ }^{8}$ Department of Electronic Science and Engineering, Kyoto University, Nishikyo-ku, Kyoto 615-8510, Japan

${ }^{9}$ Department of Physics, Ben-Gurion University of the Negev, 8410501 Beer-Sheva, Israel

${ }^{10}$ JST, PRESTO, Kawaguchi, Japan

${ }^{11}$ Quantum Information and Quantum Biology Center, Institute for Open and Transdisciplinary Research Initiatives, Osaka University, Japan

Correspondence: Koichiro Miyanishi (miyanishi@qi.mp.es.osaka-u.ac.jp), Takuya F. Segawa (segawat@ethz.ch), Makoto Negoro (negoro@qiqb.otri.osaka-u.ac.jp)

\section{Estimation of the electron spin polarization of $\mathrm{NV}^{-}$center}

Using the ESR spectrum of the nanodiamonds in Fig. 3(b), we estimated the initial electron polarization $P_{e}^{\mathrm{NV}}$ of the NV $\mathrm{NV}^{-}$center between the $m_{S}=0$ and the $m_{S}=-1$ states right after laser irradiation in the magnetic field sweep range. As the reference of the polarization, we use the thermally polarized signal from all $S=1 / 2$ electron spins which exist in the nanodiamond sample.

The electron polarization $P_{e}^{\mathrm{NV}}$ is calculated using the following equation

$P_{e}^{\mathrm{NV}}=P_{S=1 / 2} \times\left(\frac{S^{\mathrm{NV}}}{S^{S=1 / 2} \eta^{S=1 / 2}}\right) \times\left(\frac{\rho^{S=1 / 2}}{\rho_{e}^{\mathrm{NV}} \eta_{p}^{\mathrm{NV}}}\right)=0.0926 \% \times 0.47 \times\left(\frac{177 \mathrm{ppm}}{3 \mathrm{ppm} \times 0.2}\right)$,

where $P^{S=1 / 2}$ is the polarization of the spin $S=1 / 2$ in thermal equilibrium, $S^{\mathrm{NV}}$ is the area intensity of the ESR line of the $\mathrm{NV}^{-}$center integrated over the field-sweep range (corresponding to the gray area in Fig. 1), $S^{S=1 / 2}$ is the area intensity of 


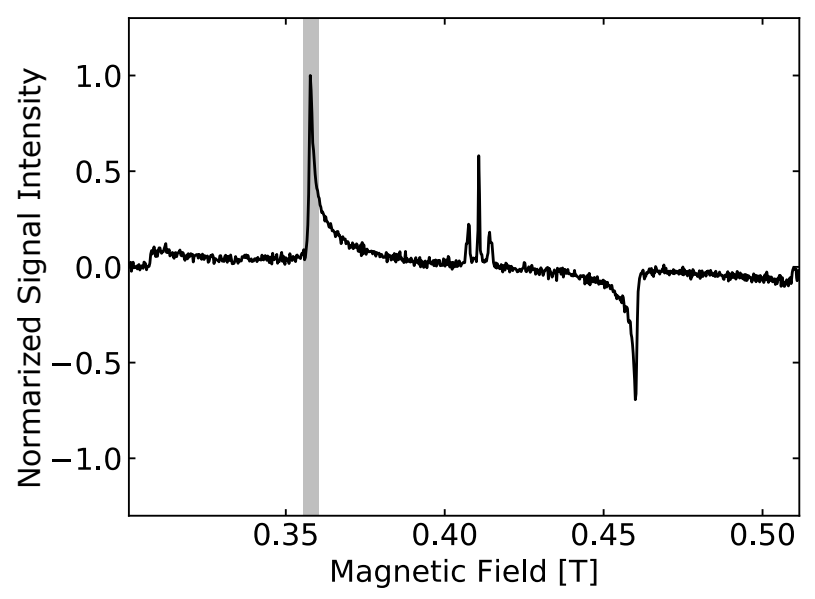

Supplementary Figure 1. ESR powder spectrum of nanodiamond samples. The gray area corresponds to the magnetic field sweep range.

the ESR line of the electron spin (corresponding to the three lines in the center of the field) center integrated over the field-

10 sweep range, $\eta^{S=1 / 2}$ is the scale factor for the signal intesity of the $S=1 / 2$ spins and $\rho^{S=1 / 2}=3.1 \times 10^{19} \mathrm{~cm}^{-3}(177 \mathrm{ppm})$ $\left(\rho_{e}^{\mathrm{NV}}=5.3 \times 10^{17} \mathrm{~cm}^{-3}(3 \mathrm{ppm})\right)$ is the density of all $S=1 / 2(\mathrm{NV})$ electron spins.

\section{NMR linewidth of ${ }^{13} \mathrm{C}$ in PBA with and without ${ }^{1} \mathrm{H}$ decoupling}

The ${ }^{13} \mathrm{C}$ NMR of PBA with and without ${ }^{1} \mathrm{H}$ decoupling is shown in Fig 2. Both signals are normalized by the maximum signal intensity. The linewidth of the ${ }^{13} \mathrm{C}$ NMR signal with ${ }^{1} \mathrm{H}$ decoupling was $1.2 \mathrm{kHz}$ and that without ${ }^{1} \mathrm{H}$ decoupling was $5.2 \mathrm{kHz}$.

15 This signal broadening is due to the dipole-dipole interaction between ${ }^{13} \mathrm{C}$ spins and ${ }^{1} \mathrm{H}$ spins, the interaction, which leads to the low spin diffusion coefficient in PBA. 


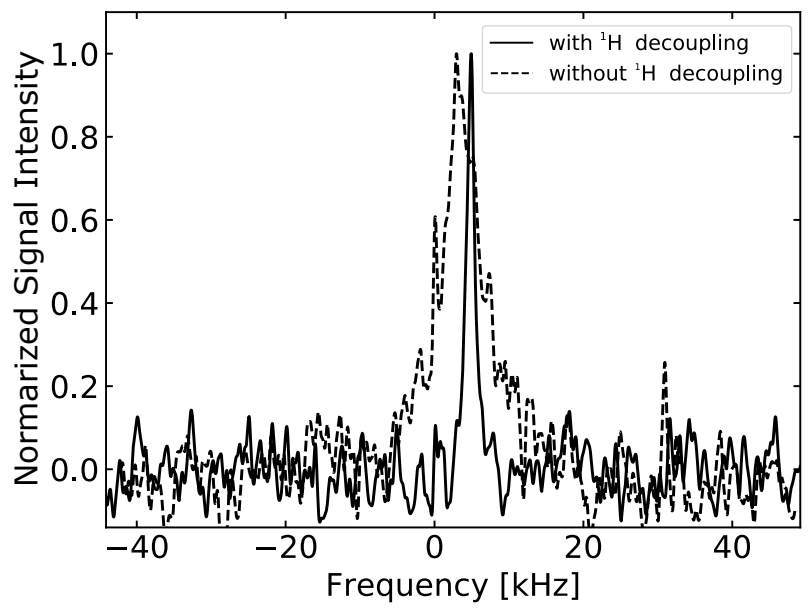

Supplementary Figure 2. NMR spectra of ${ }^{13} \mathrm{C}$ in PBA. The solid line shows the ${ }^{13} \mathrm{C}$ NMR signals with ${ }^{1} \mathrm{H}$ decoupling and the dashed line without ${ }^{1} \mathrm{H}$ decoupling.

\section{$3{ }^{13} \mathrm{C}$ hyperpolarization in microdiamonds at the 'high-field' horn}

Hyperpolarized ${ }^{13} \mathrm{C}$ NMR is performed at a magnetic field of $0.46 \mathrm{~T}$ (corresponding to the 'high-field' horn of the ESR powder spectrum of the microdiamonds in Fig. 3(d)) at a Larmor frequency of $\omega_{0, \mathrm{C}}=4.95 \mathrm{MHz}$ and a DNP-enhanced ${ }^{13} \mathrm{C}$ NMR spectrum is shown in Fig. 3(a). Buildup curves of ${ }^{13} \mathrm{C}$ polarization with laser repetition frequency of $R=30,50,70$ and $100 \mathrm{~Hz}$ are shown in Fig. 3(b).

The curve of longitudinal relaxation of the ${ }^{13} \mathrm{C}$ spins in microdiamonds are shown in Fig. 3(c) and the longitudinal relaxation time is $T_{1, \mathrm{C}}^{\mathrm{NV}}=99 \pm 14 \mathrm{~s}$. In the longitudinal relaxation measurement, we change the delay time between the DNP process and the NMR measurement. 

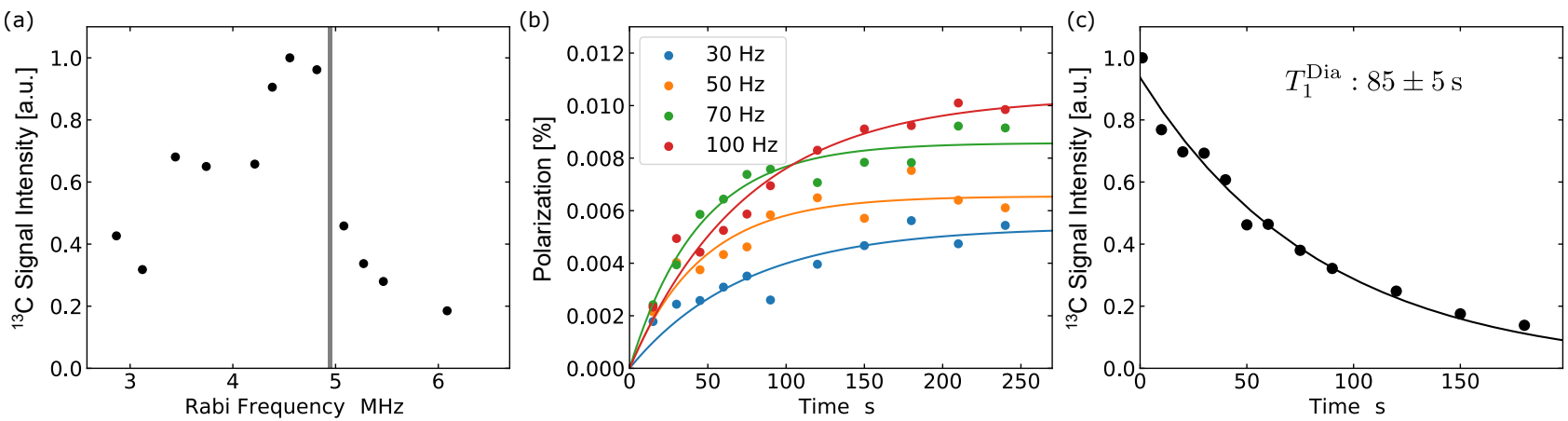

Supplementary Figure 3. Optical hyperpolarization in microdiamonds at a magnetic field of $0.46 \mathrm{~T}$ (corresponding to the 'high-field' horn of the ESR powder spectrum). (a) Signal intensity dependence on the microwave power $\omega_{\text {rabi }}^{\mathrm{MW}}$ at the high-field horn. The gray line shows the ${ }^{13} \mathrm{C}$ Larmor frequency at high-field horn. (b) Buildup curves of ${ }^{13} \mathrm{C}$ polarization in microdiamonds. The blue, orange, green and red denote the laser repetition frequency of $30 \mathrm{~Hz}, 50 \mathrm{~Hz}, 70 \mathrm{~Hz}$ and $100 \mathrm{~Hz}$. (c) The relaxation curve for the ${ }^{13} \mathrm{C}$ NMR signal of microdiamonds.

\section{Sample pictures}

The pictures of the three samples in the glass tubes are shown in Fig. 4.

Fig. 5 shows the effect of oxidation in air on the color of the nanodiamond powders. Both samples are electron irradiated and annealed $\left(800^{\circ} \mathrm{C}\right.$ in vacuum) nanodiamonds. Fig. 5(a) was taken before oxidation in air, while Fig. 5(b) was taken after oxidation in air. The oxidation procedure removes $\mathrm{sp}^{2}$ carbon on the surface of the nanodiamonds, which was created during the annealing step. The change in color from grey to white is a chemical key step to perform optical excitation of NV centers in nanodiamonds. 
(a)

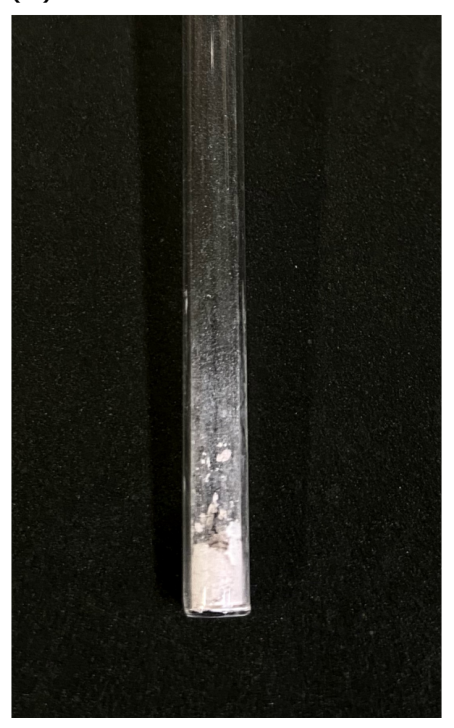

(b)

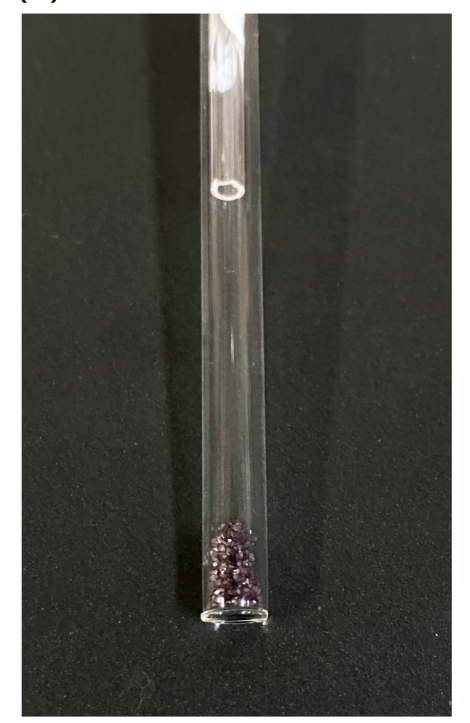

(c)

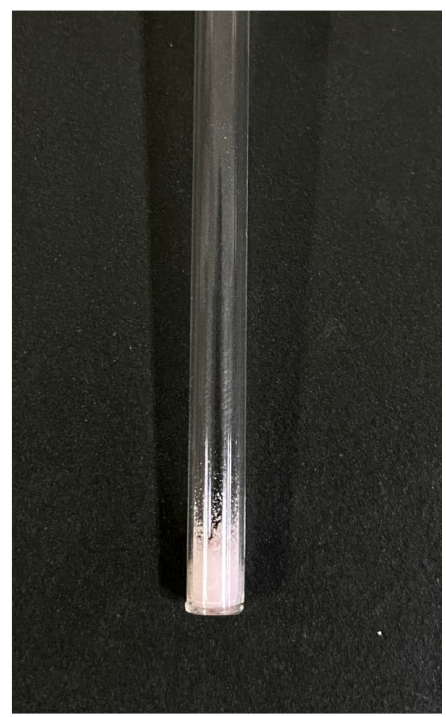

Supplementary Figure 4. Pictures of the three samples (a) nanodiamonds, (b) microdiamonds and (c) pentacene doped benzoic acid.

(a)

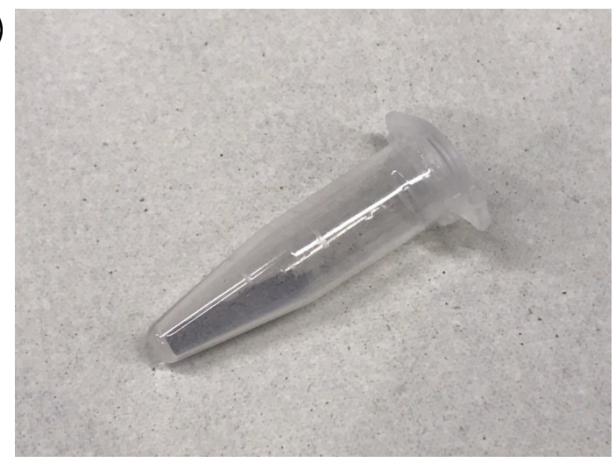

(b)

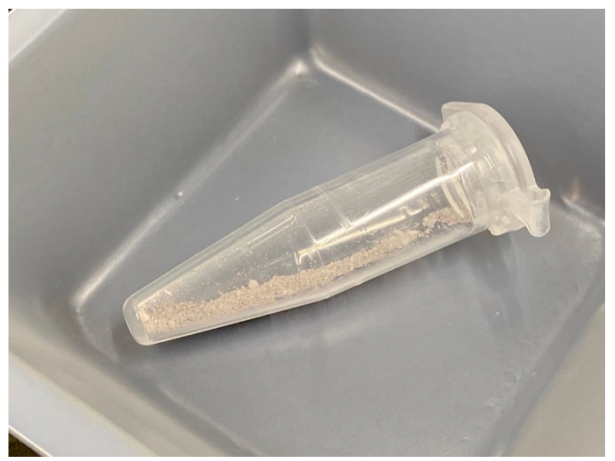

Supplementary Figure 5. Pictures of electron irradiated and annealed nanodiamonds (a) before oxidation in air ("dark") and (b) after oxidation in air ("white"). 